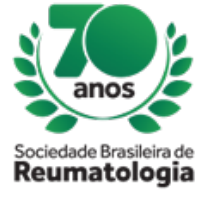

\title{
ANXIETY AND CLINICAL ASSOCIATIONS IN SYSTEMIC LUPUS ERYTHEMATOSUS PATIENTS: A CROSS- SECTIONAL STUDY IN A TERTIARY HOSPITAL FROM NORTHEAST BRAZIL
}

FLAVIA JATOBÁ BARROS (Instituto de Medicina Integral Professor Fernando Figueira - IMIP, RECIFE, PE, Brasil), BRUNA FERRAZ GUTIERREZ PIOLA (Instituto de Medicina Integral Professor Fernando Figueira IMIP, RECIFE, PE, Brasil), JOSE ANCHIETA BRITO (Faculdade Pernambucana de Saúde -FPS, RECIFE, PE, Brasil), LAURINDO FERREIRA ROCHA JR (Instituto de Medicina Integral Professor Fernando Figueira IMIP, RECIFE, PE, Brasil)

\section{BACKGROUND}

Systemic Lupus Erythematosus (SLE) is a chronic autoimmune disease that is associated with impairment of the quality of life. Neuropsychiatric disorders are common clinical manifestations of SLE, such as anxiety. This study aimed to determine the prevalence of anxiety and determine the association between anxiety and clinical parameters of SLE.

\section{MATERIALS AND METHODS}

We conducted a cross-sectional study with patients classified as having SLE according to the 1987 SLE criteria of the American College of Rheumatology (ACR) or the SLICC (Systemic Lupus Erythematosus international collaborating clinics) of 2012. Clinical and sociodemographic data was collected by interviews. For the diagnosis of anxiety, HADS-A (Hospital Anxiety and Depression Scale - anxiety) questionnaire was used. Statistical association and correlation were performed using software GraphPad prism 6.0.

\section{RESULTS}

Fifty-nine (59) patients were included in the study, of which 51 women and 8 men. The median age was 35 (range 21-71) years. Of the total, 22 patients (32.28\%) had anxiety according to de HADS-A questionnaire. The median body mass index (BMI) of the patients with anxiety was significant higher [27.80 (IQR: 24.91-31.23) vs. 23.37 (IQR: 20.37-28.71); $p=0.0477$ ], as well as the values of the visual analogic scale (VAS) for pain [35.70 (IQR: 18.80-56.98) vs. 1.500 (IQR: 0.00-38.60); $p=0.0116$, morning stiffness [50.00 (IQR: 1.810-82.80) vs. 0.0 (IQR: 0.0-45.20); $p=0.0067$ ], fatigue [86.40 (IQR: 44.18-99.25) vs. 36.30 (IQR: 2.700-54.50); $\mathrm{p}=0.0003$ ] and for patient global evaluation [92.70 (IQR: 65.50-100.0) vs. 50.00 (IQR: 21.10-91.13); $p=0.0100$ ]. In patients with anxiety the score of HADS-A had significant correlation with years of education $(p=0.0289 ; r=-0.4658)$ and with the scores of VAS for pain $(p=0.0084$; $r=0.6007)$ and for fatigue $(p=0.0161 ; r=0.5580)$. Taken all the subjects together, there were correlations with BMI ( $p=0.0325 ; r=0.2812$ ) and the scores of VAS for pain ( $p=0.0047 ; r=0.3897)$, for morning stiffness $(p=0.0035 ; r=0.3980)$, for fatigue $(p=0.0002 ; r=0.5001)$ and for patient global evaluation of disease $(p=$ $0.0035 ; r=0.3976)$.

\section{CONCLUSION}

Anxiety is present in one third of systemic lupus erythematosus patients. This condition possible affects the perception of patients about their health, as observed in the visual analogic scale scores associations found. Anxiety also is associated with body mass index. Additional studies are needed. 